\title{
El poder reunificador de la biotecnología. Reflexiones en torno a la conformación de un espacio colaborativo entre arte y ciencia'
}

Lucía Stubrin ${ }^{2}$

Universidad Nacional de Entre Ríos - Argentina

\section{Resumen}

En este artículo se buscan desplegar los replanteos epistemológicos dentro de la historia de las ciencias biológicas que prepararon el terreno para la apertura mental de los científicos y la apertura física de los laboratorios, dando lugar a la consolidación del bioarte. De esta manera, intentamos analizar el surgimiento de la biotecnología dentro de un entramado político, económico y social complejo donde los conocimientos se complementan dentro del ámbito científico, generando asimismo un

I Este artículo se desprende del proyecto de investigación doctoral titulado "Arte y ciencia: la práctica bioartística argentina en su relación con la escena internacional", realizado por la misma autora durante el período 20 I 0-20 I 4 con financiamiento del Consejo Nacional de Investigaciones Científicas y Técnicas (CONICET), y defendido con calificación 10 (Sobresaliente) y recomendación de publicación el 30 de marzo de 2015 en la Universidad de Buenos Aires, Argentina.

2 Becaria Posdoctoral del Consejo Nacional de Investigaciones Científicas y Técnicas (CONICET), Argentina; Dra. en Teoría e Historia de las Artes, Facultad de Filosofía y Letras, Universidad de Buenos Aires; Becaria Doctoral Erasmus Mundus EUROPLATA, Université de Strasbourg, Francia; Profesora de Semiótica, Universidad Nac. de Entre Ríos; Prof. de Epistemología de las Artes Visuales, Universidad Nacional del Litoral; Lic. en Comunicación Social, Universidad Nacional de Entre Ríos. Dirección electrónica: lucia.stubrin@gmail.com 
margen de conciencia sobre la falibilidad del método y la ciega especialización. El reconocimiento de las debilidades sirve como condición de posibilidad para la incorporación de actores artísticos que trabajan con los mismos materiales, desafiando las lógicas, preguntas y objetivos tradicionales de la tarea científica. El artículo, asimismo, se inscribe dentro de la investigación interdisciplinaria realizada en el marco de la tesis doctoral de quien escribe, titulada "Arte y ciencia: la práctica bioartística argentina en su relación con la escena internacional".

Palabras clave: Biotecnología, bioarte, ciencia, epistemología, arte contemporáneo, técnica, Argentina.

\title{
Re-unifying power of technology. \\ Reflections about the creation of a collaborative space between science and art
}

\begin{abstract}
This article aims at displaying the epistemological statements within the history of biological sciences which prepared the terrain for the mind opening of the scientists and physical opening of laboratories, giving place to the consolidation of bio-art. In this manner, we intend to analyze the surge of biotechnology inside a complex interwoven political, economic and social context, where knowledge is complemented in the scientific field, generating a light of consciousness about the method fertility and blind specialization. Acknowledging weaknesses serves as a feasibility condition for the involvement of artistic actors who work with the same materials, challenging the traditional logics, questions and goals of the scientific task. The article in itself belongs to the inter-disciplinary research carried out within the framework of the doctoral thesis of the undersigning, entitled "Art and Science: the Argentinean bio-artistic practice in its relation with the international scene".
\end{abstract}

Key words: bio-technology, bio-art, science, epistemology, contemporary art, technique, Argentina.

\section{$O$ poder reunificador da biotecnologia. Reflexões em torno à confirmação de um espaço colaborativo entre arte e ciência}

\section{Resumo}

Neste artigo se buscam expandir os delineamentos epistemológicos dentro da história das ciências biológicas que prepararam o terreno para a abertura mental dos cientistas e à abertura física dos laboratórios, dando lugar à consolidação da bioarte. Desta maneira, tentamos analisar o surgimento da biotecnologia dentro de um entrançado político, econômico e social complexo no qual os conhecimentos se complementam dentro do âmbito cientifico, gerando assim mesmo uma margem de consciência sobre a fiabilidade do método e a cega especialização. $\bigcirc$ reconhecimento das fraquezas 
serve como condição de possibilidade para a incorporação de atores artísticos que trabalhem com os mesmos materiais, desafiando as lógicas perguntas e objetivos tradicionais da tarefa científica. $\bigcirc$ artigo também se inscreve dentro da investigação interdisciplinar realizada no marco da tese doutoral de quem escreve, titulada "Arte e ciência: a prática bioartística argentina na sua relação com a cena internacional".

Palavras chave: biotecnologia, bioarte, ciência, epistemologia, arte contemporânea, técnica, Argentina.

\section{Introducción}

La gran pregunta que ha movilizado el universo de la biología contemporánea ha sido cómo se produce la herencia. Médicos, biólogos, físicos, ingenieros, genetistas, entre tantos otros especialistas, se vieron involucrados en el desciframiento de este acertijo que produjo, a lo largo de más de cien años, modificaciones en los modos de organización del conocimiento científico a nivel político y epistemológico, dando lugar a la aparición de nuevas disciplinas e interrogantes. Los desarrollos tecnológicos que acompañaron este proceso también tuvieron repercusiones en distintos ámbitos de la vida social complejizando la red de relaciones ente ciencia, mercado y sociedad.

El bioarte, por su parte, se inscribe en esa misma red de conocimientos técnicos, científicos, políticos, económicos y filosóficos que van más allá de su naturaleza estética. De hecho, su esencia colaborativa depende tanto del esfuerzo de los artistas que buscan experimentar con materia viva, como de los científicos que integran en sus laboratorios estos intentos interdisciplinarios.

En este sentido, es necesario que nos preguntemos cómo hemos llegado a crear las condiciones para que los ámbitos de investigación hayan abierto sus puertas a actores y experiencias tan distintas entre sí.

Para ello, identificar los principales hitos que cambiaron la manera de concebir los mecanismos mediante los cuales se produce la herencia, apunta a establecer un horizonte de comprensión donde el devenir de la investigación científica se humaniza, aceptando que los errores, los avances y retrocesos metodológicos, los descubrimientos inexplicables, etc. son parte del proceso de producción de conocimiento que no es propiedad exclusiva de ninguna esfera social en particular.

En consecuencia, la presencia del arte en los laboratorios de biotecnología, en particular, se vuelve un hecho pertinente si aceptamos a la creatividad como punto de contacto entre todas las mentes pensantes, más allá de la formación educativa que cada uno actualice en su trabajo. Destacamos el rol de la biotecnología porque su constitución histórica heterogénea e interdisciplinaria, preparó el terreno para la incorporación de artistas y se volvió el transfondo inevitable para la problematización del bioarte. 


\section{El fin del monopolio científico de lo viviente}

Eduardo Kac es uno de los principales exponentes de la línea de trabajo 'ortodoxa', dentro de las distintas visiones sobre el bioarte que existen en el amplio universo de las relaciones arte-ciencia contemporáneas. Adherente a la idea de que los artistas trabajan con los elementos de su tiempo, asume que luego de pasar por la exploración de nuevos medios como la radio, la televisión, el video, la computadora, etc., la biotecnología abre las posibilidades de experimentación con herramientas, técnicas y procedimientos de las que no podemos predecir hacia dónde nos pueden llevar.

Kac traza una distinción entre la "biotecnología tradicional" y la "biotecnología contemporánea". La primera es aquella que podemos asociar con el vino, el queso, el pan, el vinagre así como con la gran variedad de especies animales y plantas híbridas que podemos encontrar en la naturaleza, como los perros de raza y las rosas. La segunda es un poco más compleja ya que su producción no

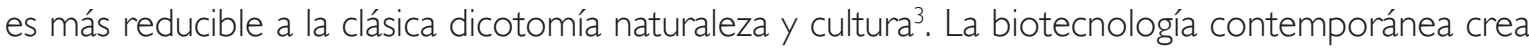
vidas nuevas así como nuevos problemas legales; afecta a la sociedad en su conjunto haciendo circular alimentos genéticamente modificados que no sabemos a ciencia cierta si son beneficiosos para la salud humana; asimismo, crea nuevos mercados; modifica los parámetros culturales de la herencia alterando la noción de identidad; opera en todos los niveles de la sociedad y por eso es muy difícil discriminar su impacto y a su vez su supuesta orientación exclusiva a la investigación científica cuando el intercambio con la producción industrial es inevitable.

En este contexto donde lo micro (los genes) puede exteriorizarse a través de la amplificación ad-infinitum de una secuencia identificada de un genoma, y lo macro (cromosoma sintético creado a partir de sustancias no vivientes) puede incorporarse a lo micro produciendo seres transgénicos, el arte no se paraliza frente al fin de las dicotomías. Por el contrario, asume el desafío de abrirse al trabajo con medios vivos y proyecta ideas y formas imposibles hasta el pasado reciente.

Desde esta perspectiva, Kac publica en 1998 el primer manifiesto -que se pueda asociar directamente al género bioartístico- al que bautizó como "Arte Transgénico". En la introducción del mismo, propone sin medias tintas que el nuevo arte debe hacer uso de las tecnologías genéticas y los implantes digitales, es decir, los últimos avances que la ciencia había hecho hasta ese momento y que afectaban no sólo la parte externa de los cuerpos que se puede advertir a partir de las cirugías plásticas y los desarrollos médicos relacionados con las neuroprótesis; sino también todo aquello que

3 García Canclini, Néstor (2004) Diferentes, desiguales y desconectados. Mapas de la interculturalidad. Barcelona. Editorial Gedisa. 2004, pág. 24: "Esta manera demasiado simple y extensa de definir la cultura, como todo lo que no es naturaleza, sirvió para distinguir lo cultural de lo biológico o genético y superar formas primarias del etnocentrismo. Ayudó a admitir como cultura lo creado por todos los hombres en todas las sociedades y en todos los tiempos. Toda sociedad tiene cultura, se decía, y por tanto no hay razones para que una discrimine o descalifique a las otras. La consecuencia política de esta definición fue el relativismo cultural: admitir que cada cultura tiene derecho a darse sus propias formas de organización y de estilos de vida, aun cuando incluyan aspectos que pueden ser sorprendentes, como los sacrificios humanos o la poligamia. Sin embargo, al abarcar con la noción de cultura tantas dimensiones de la vida social (tecnología, economía, religión, moral, arte) la noción perdía eficacia operativa. Además, se ha criticado que el reconocimiento sin jerarquías de todas las culturas como igualmente legítimas cae en una indiferenciación que las hace incomparables e inconmensurables." 
no podemos ver pero que nos afecta directamente como lo que sucede debajo de la piel y en otros organismos microscópicos y carentes de piel como las bacterias. Textualmente el artista proponía:

(...) que el arte transgénico sea una nueva forma de arte basada en el uso de las técnicas de ingeniería genética para transferir material de una especie a otra, o de crear unos singulares organismos vivientes con genes sintéticos. (...) La naturaleza de este nuevo arte no sólo es definida por el nacimiento y crecimiento de una nueva planta o un nuevo animal, sino sobretodo, por la naturaleza de la relación entre el artista, el público y el organismo transgénico. (...) No hay arte transgénico sin un compromiso firme y la aceptación de la responsabilidad por la nueva forma de vida creada. Las preocupaciones éticas son de capital importancia en cualquier obra artística y se hacen todavía más cruciales que nunca en el contexto del arte biológico, donde un ser vivo es la propia obra de arte $(. . .)^{4}$

Como mencionamos antes de la cita, dos tipos de desarrollos científicos son incorporados en el nuevo arte que plantea Kac. La combinación de tecnologías duras (hardware), blandas (software) y húmedas (biológicas), por un lado, y las tecnologías asociadas a la ingeniería genética y la microbiología en general. De acuerdo con esta cita específica del manifiesto, las obras vivas o creadas a partir de medios vivientes y manipuladas en función de las nuevas técnicas biotecnológicas forman parte de la categoría "arte transgénico". Expresamente el artista no incluye dentro de la definición otras tecnologías que no sean esas.

A pesar de que el objetivo del manifiesto de Kac es establecer un nuevo género - denominado "arte transgénico"-, nos preguntamos si en el universo del artista aparece en algún momento una propuesta sobre la definición de bioarte. Palabra que durante la década del noventa, circulaba dentro del vocabulario de las artes tecnológicas a partir de obras polémicas que se expusieron y que Kac seguramente conocía.

(...) en 199| el artista británico Marc Quinn había sorprendido con la pieza "Self', una escultura de su propia cabeza realizada con cuatro litros y medio de sangre, que se extrajo a sí mismo durante un período de cinco meses, y que debe mantenerse siempre congelada. A partir de ese momento, cada cinco años realizó una nueva escultura de su cabeza mediante el mismo procedimiento. En una entrevista realizada en el año 2000, señaló que buscaba con esa obra "documentar mi propia transformación y envejecimiento." 5

4 Kac, Eduardo. "El arte transgénico" en Leonardo Electronic Almanac. Vol. 6. N I I. California. 1998. Disponible en: http://www. ekac.org/transgenico.html Fecha de consulta: diciembre 2015.

5 Costa, Flavia y Stubrin, Lucía. "Bioarte" en Kozak, Claudia (ed.) Tecnopoéticas argentinas. Archivo blando de arte y tecnología. Buenos Aires. Caja Negra Editora. 2012, pág. 25. 
Como sucede con muchos artistas contemporáneos, la web personal es un canal de comunicación directo con sus seguidores y detractores, lo que le da un carácter dinámico y la vuelve una fuente de consulta permanente. Rastreando el sitio web de Kac (utilizado como hoja de ruta del mismo donde aparecen cronológicamente todos sus proyectos, acompañados de los documentos escritos por el propio Kac y de otros textos críticos y periodísticos que el artista selecciona para problematizar sus obras), encontramos que la palabra "bioarte" aparece por primera vez mencionada al momento de la presentación de la obra "Time Capsule", realizada en el centro cultural Casa das Rosas, en San Pablo -Brasil- en 1997 (un año antes de la publicación del manifiesto "Arte Transgénico").

Sintéticamente, en "Time Capsule" el artista se sometió a una intervención quirúrgica en la galería de arte para implantarse un chip de seguimiento, utilizado normalmente para controlar el ganado. La mención del término "bioarte" en el contexto de esta instalación hace referencia a una lectura más amplia de las obras que trabajan con material biológico pero no exclusivamente a nivel molecular. Claramente el artista pudo visualizar cuál sería la tendencia a futuro de las próximas investigaciones científicas que tendrían a la humanidad en vilo. En este sentido la combinación de tecnologías duras, blandas y húmedas era la más plausible de ser adoptada.

Un año después publicó el manifiesto y focalizó en lo "transgénico" a modo de especialización donde sólo el arte creado a partir de procedimientos de ingeniería genética puede ubicarse en esta categoría. Así, dentro del gran espectro de creación que supone el "bioarte", el "arte transgénico" sería una especie de subcategoría que siguiendo la lógica científica moderna, trabaja sólo con determinadas técnicas. Kac delimita campos de trabajo dentro del arte como la ciencia estipula límites epistemológicos entre las disciplinas y sus especialidades.

De acuerdo con la perspectiva de Kac, si una obra es transgénica, es bioartística también; pero a la inversa no se cumple: si es bioartística puede no ser necesariamente transgénica. Sin embargo, si reconocemos como tendencia la confluencia de distintos tipos de tecnologías en la era de la posbiología, entonces, debemos estar alertas ante la posibilidad de que las obras sean cada vez más difíciles de encasillar y que progresivamente el bioarte se vaya fusionando con el arte transgénico volviéndose la misma cosa.

\section{El arribo de una ciencia consciente de su interdisciplinariedad}

Los procesos históricos son determinantes en la organización y la representación del saber. La organización disciplinaria de las ciencias como nosotros la conocemos no es el simple reflejo en el saber de las divisiones naturales permanentes entre los niveles de la realidad. Es un producto histórico que, en su forma actual, se remonta al siglo XIX y al desarrollo de las universidades y las instituciones de investigación modernas.

Stefan Collini, en su introducción a Las dos culturas de Charles Percy Snow, sostiene: 
Pero la actividad social clave que planteó con una urgencia apremiante el problema de la relación de las cada vez más separadas "ciencias" con el resto de la cultura fue, desde luego, la educación. Esto se constata en todos los grandes Estados europeos, a medida que se establecían los sistemas educativos nacionales en el transcurso del siglo XIX, pero también en este caso asumió una forma particularmente aguda en Inglaterra. ${ }^{6}$

Según la definición que hemos adoptado, la biotecnología es una "actividad" que se nutre de muchas disciplinas. Por una parte, resulta difícil encasillarla como "disciplina" en sí misma dado que sus fronteras no son fáciles de delimitar; por otra parte, el lenguaje que establece varía de acuerdo al método que utiliza que no es uno solo sino varios y muy diferentes; y finalmente porque no posee una teoría que la sostenga.

Sin embargo, según Steven Shapin y Simon Schaffer, "esa cosa que se llama 'ciencia' no tiene una demarcación que se pueda tomar por una frontera natural"7. La crisis de los límites entre las disciplinas es lo que ha caracterizado a la epistemología de la segunda mitad del siglo XX y es el contexto de emergencia de la biotecnología, en el cual debería ser analizada. Desde la famosa conferencia de C. P. Snow en 1959 donde el físico reclama la reunificación del ámbito de las letras y de las ciencias en pos de una producción holista del conocimiento, hasta la "Carta de la Transdisciplinariedad" de 1994, firmada por intelectuales y científicos de las más variadas extracciones, el mundo académico se ha esforzado por reclamarse espacios de diálogo y trabajo cada vez más integrados. De ahí surgen, principalmente, tres enfoques que permiten conceptualizar los intentos científicos de trabajo mancomunado.

En primer lugar, podemos mencionar a la "multidisciplinariedad" (o "pluridisciplinariedad", como se traduciría literalmente del francés "pluridisciplinarité") que se define como la suma de miradas disciplinares diferentes sobre el mismo objeto; concierne el estudio de un objeto de una sola y misma disciplina por muchas disciplinas al mismo tiempo. La investigación multidisciplinar aporta un plus a la disciplina en cuestión, pero ese "plus" está al servicio exclusivo de esa misma disciplina. "Por ejemplo, un cuadro de Giotto puede ser estudiado por la mirada de la historia del arte cruzada con la de la física, la química, la historia de las religiones, la historia de Europa y la geometría"8. El punto de vista disciplinar sobre el objeto de estudio es profundizado y enriquecido por un aporte multidisciplinar. Es una yuxtaposición de puntos de vista especializados: la particularidad de cada uno es respetada. Los ángulos de visión son multiplicados pero cada uno sigue sus características propias (metodología, lenguaje, etc.).

6 Snow, Charles Percy. Las dos culturas [1959]. Introducción de Stefan Collini. Buenos Aires. Ediciones Nueva Visión. 2000, pág. II.

7 Shapin, Steven y Schaffer, Simon en Latour, Bruno. Nunca fuimos modernos. Ensayo de antropología simétrica. Buenos Aires. Siglo XXI. 2007, pág. 37.

8 Nicolescu, Basarab [1996] en Foucart, Jean. "Travail social et construction scientifique" en Pensée plurielle. 2008/3 n 19 , pág. 97. 
La "interdisciplinariedad", por otra parte, tiene una ambición diferente de la multidisciplinariedad. Implica la transferencia de métodos de una disciplina a la otra. Dominique Vinck afirma que existen dos maneras de practicar la interdisciplinariedad: según que los investigadores trabajen sobre un objeto común (complementariedad) o al contrario, se centren sobre una disciplina (circulación).

En el modelo de la "complementariedad", más allá de una simple yuxtaposición de aportes disciplinarios, los investigadores exploran los puntos de articulación entre sus saberes a fin de contribuir a un objetivo común: una producción conjunta, el análisis de un objeto dado o la concepción y el uso de un dispositivo experimental y de un instrumento. La complementariedad es a menudo asimétrica. En este caso, una de las disciplinas presentes juega un rol más importante que las otras. Desde este enfoque, las disciplinas vienen a transformarse las unas a las otras, a reorganizar sus campos y sus perspectivas metodológicas, así como también su epistemología.

Cuando la investigación interdisciplinar no se centra sobre un objeto o un proyecto en común, puede centrarse según el modelo de la "circulación"10 sobre una disciplina. En este caso, la investigación se polariza. Los científicos de una disciplina pueden así explorar otras para tomar prestados conceptos, métodos, interrogantes o problemas a resolver; resultados que sirven de base para nuevas preguntas. Se trata de entrar en las problemáticas y el lenguaje de las otras disciplinas para ver si ellas tienen problemas y preguntas similares y cómo las resuelven. Luego se reimportan y traducen esos préstamos.

Se pueden distinguir tres tipos de aplicaciones: a) un grado de aplicación; por ejemplo, cuando Lavoisier importa herramientas y métodos de la física experimental a la química o cuando los métodos de la física nuclear son transferidos a la medicina; b) un grado epistemológico; por ejemplo, de transferencia de la lógica formal en la epistemología del derecho; c) un grado de engendramiento de disciplinas; por ejemplo, la transferencia de métodos de la matemática al ámbito de la física engendra la física matemática.

Para poder estar en condiciones de realizar importaciones sistemáticas provenientes de otras disciplinas, el campo científico receptor debe ser capaz de administrar y soportar internamente una diversidad de competencias. Como explica Vinck,

On a alors affaires à des sciences polycompétentes et plurispécialisées autour d'un objet. De telles pratiques supposent aussi la polycompétence des chercheurs et leur capacité à situer les concepts, méthodes et données dans le champ sémantique et épistémologique de la discipline."

9 Vinck, Dominique. Pratiques de l'interdiciplinarité. Grenoble. Pub. 2000, pág. 86.

10 Op. Cit., pág. 88.

I I Vinck, Dominique [2000] en Foucart, Jean. "Travail social et construiction scientifique" en Pensée Plurielle. 2008/3. № 19, pág. 99: "Tenemos entonces asuntos de ciencias policompetentes y pluriespecializadas en torno de un objeto. Tales prácticas suponen también la policompetencia de los investigadores y su capacidad para situar los conceptos, métodos y datos en el campo semántico y epistemológico de la disciplina" (Traducción propia) 
La "transdisciplina" por su parte concierne, como el prefijo "trans" lo indica, aquello que está a la vez entre las disciplinas, a través de las diferentes disciplinas y más allá de toda disciplina. Es complementaria del enfoque disciplinario; hace emerger de la confrontación de disciplinas, nuevos datos que las articulan entre sí, y nos ofrece una nueva visión de la realidad. No busca la matriz de varias disciplinas, sino la apertura de todas a eso que las atraviesa y las sobrepasa. En resumen, "un discours multidimenssional non totalitaire, théorique mais non doctrinal"'2.

Explicado de otro modo, la transdisciplina tiene en cuenta las consecuencias de un flujo de información circulante de una rama del conocimiento a otra, permitiendo la emergencia de la unidad en la diversidad y de la diversidad en la unidad. Su objetivo es poner al desnudo la naturaleza y las características de ese flujo de información y su tarea prioritaria consiste en la elaboración de un nuevo lenguaje, de una nueva lógica, de nuevos conceptos para permitir la emergencia de un verdadero diálogo entre los especialistas de las diferentes áreas del conocimiento.

Sin llegar necesariamente a la creación de una nueva disciplina (como sí sugiere Legendre, por ejemplo) ${ }^{13}$, la transdisciplina se define generalmente como la integración y la transformación de campos de conocimiento con el objetivo de plantear, abordar y resolver los problemas complejos de nuestro mundo.

Ainsi, alors que la recherche disciplinaire explore un seul niveau de réalité, la recherche transdisciplinaire s'intéresse à la dynamique engendrée par l'action de plusieurs niveaux de réalité à la fois. ${ }^{14}$

Lógicamente, el descubrimiento de esta dinámica debuta con el conocimiento disciplinar, que permite constatar que, lejos de estar enfrentadas, la investigación disciplinaria, interdisciplinaria y transdisciplinaria se complementan.

La biotecnología, entonces, resulta de la complementación entre las nuevas técnicas encabezadas por la ingeniería genética y la aplicación práctica de los avances científicos en materia de ciencias biológicas. Cuando decimos "aplicación práctica" nos referimos a la posibilidad de volcar a la sociedad, mediante la industria y el mercado, los descubrimientos que puedan traducirse en procesos de producción de alimentos, fármacos, etc.

12 Lemoigne [1990] en Foucart, Jean. "Travail social et construiction scientifique" en Pensée Plurielle. 2008/3. N 19, pág. 99: "Un discurso multidimensional no totalitario, teórico pero no doctrinal." (Traducción propia).

13 Op. Cit. Según Foucart: "Legendre (1988) pousse le résultat de cette interpénétration jusqu'à la création même de cette nouvelle discipline. II définit ainsi la recherche transdisciplinaire : 'l'étude d'un problème ou d'un objet par des spécialistes divers qui situent leurs réflexions au-delà de leurs domaines respectifs et des intersections entre eux, en vue de la fusion des savoirs et des approches conduisant à l'émergence d'une nouvelle discipline'." (Foucart, 2008: 100).

"Legendre (1988) presiona el resultado de la interpenetración hasta la creación misma de una nueva disciplina. Define la investigación transdisciplinaria como: 'el estudio de un problema o un objeto por diversos especialistas que sitúan sus pensamientos más allá de sus respectivas áreas e intersecciones entre ellas, con vistas a la fusión de conocimientos y enfoques que conducen a la emergencia de una nueva disciplina."' (Traducción propia).

I4 Nicolescu, Basarab en Op. Cit., pág. 100: "Así, mientras que la investigación disciplinaria explora un solo nivel de realidad, la investigación transdisciplinaria se interesa en la dinámica engendrada por la acción de muchos niveles de realidad al mismo tiempo." (Traducción propia). 
Así establecida, pareciera que la biotecnología no tuviera objetivos epistemológicos en sí misma que la ubicaran dentro del ámbito de las disciplinas científicas. Sin embargo, como hemos visto a lo largo de la marcha de las ciencias de la vida durante el siglo $X X$, el entrecruzamiento entre distintas miradas como la de la física, la química, la medicina, la matemática y la biología, fue la razón del avance más grande que se ha producido en su historia. Lo que demuestra también la teoría kunhiana de que no es el método, como pretendía Karl Popper, el que define a una ciencia sino el consenso científico dentro del paradigma.

En La estructura de las revoluciones científicas (1962), Thomas Kuhn afirma que el progreso de la ciencia no se debe a la aplicación del método científico sino al acuerdo de la comunidad científica en la elección de una teoría (que no necesariamente debe resolver todos los interrogantes del campo) que se erigirá en paradigma durante un tiempo. En este sentido, desde el diseño estadístico mendeliano, pasando por la fisiología de Morgan hasta la instalación de la perspectiva molecular para el análisis de los mecanismos de la herencia, los métodos han sido justamente el indicio del cambio, como consecuencia de las transformaciones en la perspectiva de la comunidad de miembros autorizados. Lo que diferencia a la biotecnología es su capacidad de reunión de todos esos conocimientos científicos y tecnológicos, sin prejuicios, y al servicio de una transferencia que no por ser concreta resulta menos valiosa a nivel epistemológico.

La existencia misma de la ciencia depende de que el poder de escoger entre paradigmas se delegue en los miembros de una comunidad de tipo especial. (...) Los miembros del grupo, como individuos y en virtud de su preparación y la experiencia que comparten, deberán ser considerados como los únicos poseedores de las reglas del juego o de alguna base equivalente para emitir juicios inequívocos. ${ }^{15}$

El aporte de Kuhn nos permite abrir el panorama en lo que respecta a las fuentes de producción de conocimiento. Poner en evidencia el poder que la comunidad científica tiene en la definición de lo que es ciencia coincide, a su vez, con la división histórico-política de compartimentación del conocimiento y su moderna búsqueda de la especialización educativa que plantea Snow.

Se podría decir que la biotecnología es una disciplina consciente de su condición interdisciplinaria de tipo "complementaria", retomando a Vinck, dado que está integrada por una comunidad de saberes variados que se combinan para alcanzar un objetivo común, relacionado directamente con los intereses de la sociedad. Asimismo, la crisis de autoridad que implícitamente rodea a la comunidad científica alimentada por el autoconocimiento de sus propias limitaciones -reconocida por caso en la Conferencia Asilomar- y la falacia del poder de la "bata blanca" como fuente última de conocimiento, permiten incorporar poco a poco saberes no articulados de disciplinas y ámbitos totalmente diversos (por ejemplo, de la esfera artística).

I5 Kuhn, Thomas. Las estructura de las revoluciones científicas. México. Fondo de Cultura Económica. 1986. págs. 258-259. 
Si la historia de la biología en el siglo XX se ha presentado como una sucesión de giros epistemológicos donde diferentes perspectivas - medicina, química, física, matemática- ${ }^{16}$ iban reemplazándose en forma lineal, la biotecnología es la síntesis sincrónica de este proceso que comprime los saberes, valiéndose de aquellos que le son más útiles en función de la circunstancia y no del dogma científico establecido.

En Argentina, las carreras de Licenciatura en Biotecnología son de cinco años aproximadamente y se pueden cursar en universidades públicas y privadas. El perfil del egresado, según la Facultad de Bioquímica y Ciencias Biológicas de la Universidad Nacional del Litoral, sostiene que:

Puede encargarse de la planificación y el desarrollo de los procesos biotecnológicos llevados a cabo en laboratorios, plantas piloto o industriales, como así también del control de calidad de los insumos y productos empleados en ellos. Puede desarrollar y dirigir procesos de producción que impliquen el uso de microorganismos, cultivos celulares u otras moléculas de origen biológico y sintético; desarrollar procedimientos, reactivos y sistemas de diagnóstico que permitan determinar enfermedades que afectan la salud humana, animal y vegetal; como así también procesos biológicos para el control y descontaminación de efluentes y para la preservación del medio ambiente. Está capacitado para realizar tareas de investigación, especialmente en el campo de los bioprocesos, la genética, la biología molecular y celular, entre otros; realizar asesoramientos técnicos y científicos sobre la valorización de recursos biológicos y bioquímicos aprovechables; realizar relevamientos, asesoramientos y peritajes técnicos y científicos en áreas de la biología y la microbiología a nivel molecular y celular. ${ }^{17}$

Podríamos señalar, en este sentido, que se trata de una disciplina interdisciplinaria y pragmática, dado que su naturaleza "fronteriza", o como dirían Star y Griesemer a través de Robert Bud en tanto "boundary object", permite que se acomode fácilmente a los cambios tecnológicos y a las regulaciones que acompañan la relación ciencia-sociedad. La paleta de saberes de la que se nutre la biotecnología es muy amplia y diversa lo que genera que un biotecnólogo sea varios profesionales a la vez. Su mayor fortaleza reside, justamente, en la habilidad para identificar el momento y la manera en que deben aplicar el conocimiento apropiado.

La variedad de líneas de trabajo que comprende su formación puede ser también una fuente de conflicto. Sobre todo, en materia de legislación, ésta debe acompañar rápidamente los cambios

16 Sostiene Abir-Am, Pnina Geraldine. "La biología molecular en el contexto de las culturas del Reino Unido, Francia y Estados Unidos" en La ciencia y sus culturas. Revista Internacional de Ciencias Sociales. Junio 200 I. № I68. Organización de Estados Americanos. Disponible en: http://www.oei.es/salactsi/abir.pdf: "Como megadisciplina nueva, integradora, interdisciplinaria e internacional, con un impacto sobre la sociedad de gran alcance y en continuo crecimiento, la biología molecular se ha desarrollado históricamente en tres grandes etapas, cada una de ellas bajo la influencia fundamental de una de las ciencias exactas: la química, la física y las matemáticas (Abir-Am 1992/3, 1997; Morange 1998; De Chadarevian y Kamminga (eds.) 1998)."

17 Facultad de Bioquímica y Ciencias Biológicas - Universidad Nacional del Litoral (20I4) Diponible en: http://www.fbcb.unl.edu. ar/pages/estudios/carreras-de-grado/lic.-en-biotecnologia.php Fecha de consulta: diciembre 2015. 
que se producen a nivel epistemológico a partir de las metodologías y resultados innovadores que se obtienen permanentemente en la investigación.

Por ejemplo, el acuerdo Bayh-Dole fue un intento de regulación del intercambio de conocimientos entre el ámbito público y privado que instaló en la opinión pública los dilemas de la ciencia tanto en su dimensión ética como mercantil. Su aparición devino en un elemento central para el armado del complejo paisaje social al que nos ha llevado la ciencia interdisciplinaria contemporánea y todos sus actores involucrados en el proceso. A continuación desarrollaremos las circunstancias en las que se produjo este acuerdo tan importante para la consolidación de un sistema de funcionamiento de la biotecnología a nivel global.

\section{Transfondo histórico-político de un cambio de paradigma}

Veinticinco años después de Asilomar, los científicos se preguntaban -en una nueva edición de la conferencia realizada en el año 2000 - si ese acto de arrojo que habían tenido en 1975, cuando habían decidido solos detener su propia actividad, convenía mantenerlo en el nuevo milenio también. En el pasado, habían reclamado autonomía para hacerse cargo del control y del establecimiento de las reglas de bioseguridad. En primer lugar, con el objetivo de proteger la vida de quienes estaban experimentando, y en segundo lugar, preocupados por la reacción social de la comunidad.

Si bien en aquel momento los riegos se vislumbraban altos -aunque en gran medida también desconocidos, las potencialidades de las nuevas técnicas eran mayores y la ciencia no podía permitirse que la amenaza del azar minara las posibilidades de la investigación mediante una sanción social definitiva.

Los científicos, entonces, se hicieron cargo para protegerse y para salvarse, ya que su tarea podía ser suspendida debido a resistencias políticas heredadas de los tratamientos eugenésicos realizados durante el nazismo, el precedente de la bomba atómica y la guerra de Vietnam, junto con la desconfianza, el miedo y el desconcierto generalizado que suscitaban las nuevas técnicas y la ciencia en general en el contexto de la guerra fría' ${ }^{18}$.

I8 En noviembre de 1970 se realizó en Londres una reunión organizada por la British Society for Social Responsibility in Science (BSSRS) para discutir el Impacto Social de la Biología Moderna (Social Impact of Modern Biology), a la que asistieron un promedio de 700 científicos de distintas categorías durante los tres días que duró. La sesión inaugural marcó el eje central del congreso. Las palabras estuvieron a cargo del científico Maurice Wilkins (biofísico: participó del Proyecto Manhatan y en 1962 recibió el Premio Nobel junto a Francis Crick y James Watson por sus descubrimientos referentes a la estructura molecular del ADN). Wilkins sostuvo: "We have to face the fact that there is a crisis on science today (...) The main cause is probably the Bomb: scientists no longer have their almost arrogant condence in the value of science. At the same time non-scientists increasingly and openly question the value of science. There are extremists who go further and object to rational thought as a whole." Agar, Jon. "What happened in the sixties?" en The British Journal for the History of Science. 4 I (04). 2008. P. 57I. Disponible en: http:// www.ucl.ac.uk/sts/staff/agar/documents/whathappenedinthesixties.pdf Fecha de consulta: diciembre 20 I 5.

"Tenemos que enfrentar el hecho de que hay una crisis en la ciencia hoy en día (...) La principal causa es probablemente la bomba: los científicos ya no tienen su casi arrogante confianza en el valor de la ciencia. Al mismo tiempo no-científicos cuestionan cada vez más y abiertamente el valor de la ciencia. Hay extremistas que van más allá y objetan el pensamiento racional en su conjunto." (Nuestra traducción). 
Les organisateurs décidèrent de ne pas aborder les questions éthiques touchant aux modifications génétiques et de s'en tenir aux questions de sécurité qu'ils pensaient pouvoir aborder en tant que scientifiques. (...) Ces directives ne permettaient pas seulement aux recherches de se poursuivre, mais aussi de persuader le Congrès américain que des lois restrictives n'étaient pas nécessaires. Bref, que les scientifiques pouvaient se gouverner eux-mêmes. ${ }^{19}$

Sin embargo, la expansión de la biotecnología a todos los ámbitos de la vida extendió el ámbito de debate también, haciendo que se instale la duda sobre el exclusivo control científico de su devenir. Lo que en 1975 parecía todavía ciencia ficción, fue progresivamente banalizándose y ha proporcionado lo que David Baltimore (co-organizador de Asilomar) llama "una cosecha remarcable"20 de productos y aplicaciones, incluyendo los cultivos genéticamente modificados-OGM: organismos genéticamente modificados-, los exámenes de enfermedades genéticas y la terapia génica en humanos.

La reedición de la conferencia Asilomar en el año 2000, encuentra a la comunidad científica dentro de un contexto social totalmente distinto donde el público prevalece intranquilo y atento en cuanto a las aplicaciones de la ingeniería genética. Las grandes manifestaciones contra los OGM en Europa son un claro ejemplo de la voluntad de formar parte de la discusión que tiene la sociedad. "No existen riesgos importantes que los científicos puedan asumir solos", afirma Harold Shapiro, Rector de la Universidad de Princeton y de la Comisión Consultativa Nacional de Bioética: "Los científicos pueden aportar mucho pero no pueden decidir solos"21, reitera.

Asimismo, la relación entre la investigación en biotecnología y la industria ha hecho que las universidades formen parte del entramado económico donde se comercializan los productos y tratamientos genéticos. De los orígenes puramente académicos de la primera conferencia Asilomar ya no quedan resabios porque la comunidad científica ha cambiado. Actualmente, la mayoría de los investigadores universitarios "seniors" tienen vínculos con las sociedades de biotecnología (que pueden ser privadas, públicas o a veces mixtas como las empresas incubadas en universidades públicas), lo que complica todo atisbo de auto análisis.

El fenómeno de trabajo conjunto entre científicos y el mercado ha sido en gran medida habilitado por la Ley Bayh-Dole de 1980, así como por las características propias de la epistemología biotecnológica. Desde una perspectiva histórica, el acuerdo fue consensuado entre los dos partidos mayoritarios de EEUU, con el objetivo de sacar adelante la crisis de innovación del sistema científico

19 Barinaga, Marcia. "Asilomar, vingt-cinq ans après" en La Recherche. L'actualité des sciences. No 332. P. 82. 01/06/2000. Disponible en: http://www.larecherche.fr/idees/livres/asilomar-vingt-cinq-ans-apres-0 I-06-2000-86943 Fecha de consulta: diciembre 20 I 5: "Los organizadores decidieron no abordar las cuestiones éticas relacionadas con los cambios genéticos y limitarse a las cuestiones de seguridad que pensaban podían abordar en tanto que científicos. (...) Estas directrices no sólo permitieron que la investigación continuara, sino también persuadir al Congreso de EE.UU. de que leyes restrictivas no eran necesarias. En resumen, que los científicos podían gobernarse ellos mismos." (Nuestra traducción).

20 Idem.

21 Idem. 
norteamericano, amenazado por el desarrollo de Japón y de Alemania Occidental. El nombre de la ley tiene su origen en la firma del senador demócrata -Birch Bayh-y del senador republicano -Bob Dole-, encargados de liderar el proceso político hasta la sanción del acta.

Por otra parte, la integración de procesos orgánicos dentro de la estructura industrial -que es parte de la tarea y perfil profesional de los biotecnólogos- también contribuyó a crear un contexto epistémico apropiado para que el acuerdo político tuviera éxito en su aplicación. La articulación de conocimientos teóricos y prácticos es una característica propia de la biotecnología, y un elemento que la distingue de otro tipo de científicos como aquellos que se dedican a la biología molecular -orientada al estudio de mapas genéticos incompletos, por ejemplo- o a la ingeniería genética-vinculada al desarrollo de nuevas técnicas para la manipulación de vida-. En estos casos, tanto los biólogos como los ingenieros, poseen objetivos que distan de las demandas directas del mercado y, en consecuencia, producen conocimiento en forma un poco más independiente de los centros financieros que controlan la producción de bienes y servicios pertenecientes al sector de alimentos y de salud, principalmente.

Si focalizamos nuevamente en la relación arte-ciencia que reactualiza la biotecnología, constatamos que Robert Mitchell, por ejemplo, es uno de los que prefiere resaltar los cambios en la legislación entre empresas y el sistema científico público de EEUU como un elemento fundamental de la existencia del bioarte. El acuerdo Bayh-Dole firmado por el congreso norteamericano, instaló una nueva ecología de la innovación ("innovation ecology"). Este acta significó el comienzo del trabajo "mancomunado" entre la ciencia y las corporaciones. Lo que la ecología de la innovación propone es que los ciudadanos comunes a través de sus impuestos, sus conocimientos y su predisposición a donar muestras de material vivo contribuyan a la investigación de las universidades públicas; luego los descubrimientos básicos generados en estos ámbitos son transferidos al ámbito privado a través del sistema de patentes; las empresas adquieren las patentes y producen a gran escala drogas y tratamientos que por la competencia que generan vuelven al público en forma de mercancías que intercambian por dinero.

La nueva ecología estrecha los vínculos entre el ámbito científico y el privado o, al menos, introduce una forma distinta de relacionamiento. Luego de varias décadas de sancionado el acuerdo Bayh-Dole todavía es difícil hacer funcionar esta lógica -que se ha exportado a todo el mundo- por varias razones. En primer lugar porque es necesario encontrar una herramienta, técnica o práctica que sea de interés desarrollar para los dos ámbitos. En segundo lugar porque, una vez conseguido este acuerdo, nada garantiza que sea duradero en el tiempo dado que las lógicas de mercado son distintas a las lógicas de la investigación académica. Esto significa que existe una tensión permanente entre las dos esferas que hace que, por un lado se borren temporalmente los límites que las separan, $y$, por otro lado, se pierdan las garantías de un interés a largo plazo haciendo que se refunden permanentemente los caminos a recorrer para la continuación o el fin de esta sociedad.

En relación con el corrimiento de las fronteras entre la ciencia y las corporaciones, los críticos del Bayh-Dole Act plantean que este acuerdo ha generado gran confusión en la dinámica de cada ámbito haciendo que los científicos, por ejemplo, no compartan sus conocimientos con otros investigadores porque prefieren ser dueños de patentes. En este sentido, se vuelven más reticentes a intercambiar 
gratuitamente sus descubrimientos y materiales, dificultando el libre flujo de información del que se supone depende la esfera científica.

Por otra parte, es evidente cómo este acuerdo ha favorecido el crecimiento de algunas áreas de la investigación. Específicamente el área de la salud.

Within the system of academic research, for example, the Bayh-Dole Act has arguably altered the practices of biologists working on human genetics far more than it has altered the practices of, say, the E. cole research community. (Mitchell, 2010: $58)^{22}$

Otro de los argumentos de quienes se oponen al acuerdo, reside en que los ciudadanos se vuelven consumidores del sistema de salud alimentado por las corporaciones, en lugar de que el Estado asuma la responsabilidad de proveer de salud pública a toda su población.

En este entramado de nuevas relaciones el bioarte se ubica, para Mitchell, en el medio y no, como otros autores opinan, fuera del espacio de tensión entre la ciencia y el capital. Frente a los cambios sociales que instaura la biotecnología, existen interpretaciones que pretenden ubicar al bioarte en un lugar de objetividad por su distancia crítica respecto de los objetos en disputa: conocimiento científico y negocios. Mitchell prefiere pensar al bioarte como un actor que opera dentro de la misma lógica pero generando sus propios flujos de información, dinero y materiales.

Resulta ingenuo pensar que el bioarte no tiene intereses propios o posiciones tomadas frente a la problemática de la biotecnología. Es difícil pensar que los bioartistas no están de acuerdo con el desarrollo de una "ecología de la innovación" cuando sus obras necesitan de un intercambio de conocimientos con el ámbito científico así como formas de financiamiento que permitan la experimentación y la creación con material viviente. Lo que sí podemos arriesgar es que lo que no están dispuestos a aceptar es que estos cambios sean establecidos sólo entre los actores que define el acuerdo Bayh-Dole.

En este contexto resultan pertinentes las reflexiones del filósofo Vilém Flusser-cuyas ideas han sido fuente de inspiración de Eduardo Kac, en lo que respecta a arrebatarle a la ciencia el poder de decisión, sobre todo, cuando se trata de la manipulación de vida.

(...) queda claro que no es posible abandonar la biotecnología a los técnicos, y que es preciso que los artistas participen de la aventura. El desafío es obvio: disponemos actualmente de la técnica (arte) capaz de crear no sólo seres vivos sino también formas de vida con procesos mentales ("espíritu") nuevos. Disponemos actualmente de la técnica (arte) apta para crear algo hasta ahora inimaginado e inimaginable:

22 Mitchell, Robert. Bioart and the vitality of media. Seattle. University of Washington Press, pág. 58: "Dentro del sistema de investigación académica, por ejemplo, la Ley Bayh-Dole ha alterado sin dudas las prácticas de los biólogos que trabajan en genética humana mucho más de lo que ha alterado las prácticas de, por ejemplo, la comunidad de investigadores — de la bacteria— E. cole." (Traducción propia). 
un espíritu vivo nuevo. Espíritu éste cuyo propio creador será incapaz de comprender, ya que estará basado en información genética que no es la propia. Esta es una tarea no para biotecnólogos abandonados a su propia disciplina sino para artistas en colaboración con los laboratorios actualmente establecidos. En rigor, las escuelas de arte deberían mudarse a esos laboratorios, y los laboratorios deberían formar parte de las escuelas de arte. $^{23}$

El arte puede producir diálogos entre variados sponsors de la "ecología de la innovación" y entre diferentes públicos que no están directamente involucrados con la práctica biotecnológica pero que sí se ven afectados por sus consecuencias y no saben o no encuentran espacios para entrar en contacto con el debate. Al respecto, Mitchell y Flusser comparten la creencia de la fortaleza que supone para el bioarte operar desde dentro de la misma lógica que define las nuevas relaciones entre ciencia, arte y mercado.

From this perspective, the fact that bioartists are themselves interested parties, that the desires that motivate their projects are drawn from this field, and that their vision of a public sphere seems tied to this ecology are not necessarily intractable problems or evidence of bad faith on the part of the artists. Rather, these interests, desires, and goals can serve as the vectors through which vitalist bioart produces new folds. ${ }^{24}$

La naturaleza interdisciplinaria de la biotecnología supone un gran paso en este sentido. Es por ello que, como dice Flusser, ésta no puede abandonarse a los técnicos, por lo que es necesario que se abra un poco más todavía a lo que él llama "conocimiento no articulado".

No discutiré el nebuloso término de creación, pero recordaré el approach informático que sugiere que la información nueva es creada por la introducción de ruidos en informaciones redundantes. Esto es: lo nuevo se crea al abrirse lo viejo a lo todavía no articulado. En este sentido, no hay diferencia entre la creación en ciencia y en arte. Los científicos siempre han estado abiertos a vivencias no articuladas y los artistas a conocimientos no articulados. Toda creación científica es "obra de arte", toda creación artística es "articulación de conocimiento". Por ejemplo: es fácil mostrar la vivencia barroca en el sistema de Newton, la romántica en el sistema de Darwin, la geometría perspectivista en las pinturas renacentistas y la matemática de conjuntos en la composición de Schoenberg. Es necesario llevar al nivel de la conciencia tal conexión subterránea que siempre ha unido ciencia y arte. Esa co-

23 Flusser, Vilém. "Arte vivo" [1988] en Artefacto. Pensamientos sobre la técnica. № 6. Buenos Aires: edición independiente. 2007, págs. 79-80. Disponible en: http://www.revista-artefacto.com.ar/pdf_notas/I 62.pdf Fecha de consulta: diciembre 20 I 5.

24 Mitchell, Robert. Bioart and the vitality of media. Seattle. University of Washington Press, pág. 62: "Desde esta perspectiva, el hecho de que los bioartistas sean partes interesadas, que los deseos que motivan sus proyectos sean diseñados desde el mismo campo, y que su visión de una esfera pública aparezca ligada a esta ecología no son necesariamente problemas intratables o evidencias de futuros fracasos por parte de los artistas. Por el contrario, estos intereses, deseos y objetivos pueden servir de vectores mediante los cuales el bioarte produce nuevos pliegues." (Traducción propia). 
nexión interrumpida entre vivencia y conocimiento debe ser hecha consciente si queremos tener vivencias y conocimientos plenamente humanos, esto es, políticos, intersubjetivos. ${ }^{25}$

\section{Arte y ciencia unidos por la indeterminación metodológica}

La coexistencia de perspectivas es una característica con la que los científicos han tenido que aprender a convivir. Esto es consecuencia de una silenciosa resignación que asume las limitaciones humanas en la interpretación de las supuestas leyes físicas, al mismo tiempo que reconoce la ausencia de un método basado en la causalidad que pueda explicarlo todo y al que se pueda acudir siempre para corroborar un descubrimiento.

El surgimiento de, al menos, dos niveles de Realidad diferentes en el estudio de los sistemas naturales es un acontecimiento capital en la historia del conocimiento. Puede llevarnos a repensar nuestra vida individual y social, a dar una nueva lectura a los conocimientos antiguos, a explorar de otra manera el conocimiento de nosotros mismos aquí y ahora. ${ }^{26}$

El caballito de batalla de las ciencias ha sido siempre la defensa de un método capaz de garantizar las condiciones de validez del conocimiento. Este pensamiento que nace en la física se adopta en todos los campos del saber y poco a poco va demostrando sus falencias que, aunque manifiestas en el ámbito de algunas corrientes de filosofía contemporánea, ${ }^{27}$ resultan más difíciles de asumir en otros dominios, sobre todo, en aquellos relacionados con las ciencias experimentales.

Resulta necesario destacar como factor determinante del nacimiento de la biotecnología, el predominio de la ciencia física hasta el siglo XIX y el progresivo desplazamiento de la misma a lo largo del siglo $X X$ por las ciencias biológicas. No sólo fue esta última la que modificó su modo de producción de conocimiento obteniendo un nuevo protagonismo, sino que la primera también sufrió cambios importantes que alteraron el paradigma clásico en el que se desarrolló y se sostuvo desde Galileo. El doble movimiento de retroceso, si se quiere, de la física y de avance de la biología (que ya hemos desarrollado) tiene su fundamento en el descubrimiento de la física cuántica. El elemento clave de la nueva teoría fue la inauguración de la conciencia de la existencia de distintos niveles de realidad.

25 Flusser, Vilém. "Creación científica y artística" [1982] en Artefacto. Pensamiento sobre la técnica. № 6. Buenos Aires: edición independiente. 2007, pág.77. Disponible en: http://www.revista-artefacto.com.ar/pdf_notas/ I 62.pdfFecha de consulta: diciembre 2015.

26 Op.Cit., pág. 24.

27 Op. Cit., pág. 24. Por ejemplo, amplía Nicolescu: "En nuestro siglo, Husserl y otros investigadores, en un esfuerzo por interrogarse sobre los fundamentos de la ciencia, descubrieron la existencia de los diferentes niveles de percepción de la Realidad por el sujeto-observador. Pero fueron marginados por los filósofos académicos e incomprendidos por los físicos, encerrados en su propia especialidad. De hecho, ellos fueron los pioneros de la exploración de una realidad multidimensional y multireferencial donde el ser humano puede encontrar su lugar y su verticalidad." 
Esto produjo la crisis de la ideología cientificista y la ilusión de la objetividad basada en el método.

Hasta el establecimiento del "quantum" (umbrales del siglo XX), la física podía establecer la distancia entre dos partículas u ondas a un nivel macromolecular. Basado en un método matemático, los puntos de origen y de destino conocidos permitían definir la trayectoria que los unía y, en caso de no conocer alguna de las partes de la ecuación, mediante razonamientos lógico-deductivos, se podía suplantar la ausencia. La física clásica estaba sostenida por la idea de "continuidad". Dos puntos separados por distancias infinitas están ligados por un encadenamiento de causas y efectos locales que unen todo lo conocido. El "determinismo" también tiene su justificación en la física clásica dado que ante el conocimiento de las condiciones iniciales del objeto (posición y velocidad) se puede predecir el estado físico en cualquier momento dado del tiempo.

De esta manera la física clásica fue considerada el ejemplo de ciencia moderna ya que cumplía con los postulados establecidos al momento de la ruptura con la tradición anterior, donde la división sujeto-objeto no existía como parte de la cosmovisión.

(...) la ciencia moderna proporcionaba tres postulados fundamentales, que prolongaban, en grado supremo sobre el plano de la razón, la búsqueda de leyes y de orden:

I. La existencia de las leyes universales, de carácter matemático.

2. El descubrimiento de estas leyes por medio de la experiencia científica.

3. La reproductividad perfecta de los datos experimentales. ${ }^{28}$

El paradigma científico moderno se extendió a todas las ramas de la investigación, incluyendo las ciencias sociales y humanas en general. Desde el punto de vista de Nicolescu, la teoría marxiana, por ejemplo, es un claro caso de determinismo enraizado en una idea lineal de tiempo donde las transformaciones sociales se producen a partir de una relación causal entre la base y la superestructura. ${ }^{29}$

La aparición a comienzos del siglo XX de la teoría cuántica de Max Planck tuvo consecuencias irreversibles en el pensamiento científico y cultural dado que puso de manifiesto la existencia de la discontinuidad en el tiempo, es decir, la necesidad de concebir que entre dos puntos puede existir la nada. Como expresa Nicolescu: "cuestionar la continuidad corresponde a cuestionar la causalidad local y abrir, así, una caja de Pandora"30.

Para la física, el mundo del quantum funciona con otra lógica que es la del "indeterminismo", esto no significa que tenga un funcionamiento azaroso, sino que no se corresponde con el modelo

28 Nicolescu, Basarab. La Transdisciplinariedad. Manifiesto. México. Edición: 7 Saberes. 1996, pág. 16.

29 Op. Cit., pág. I 8: "En la euforia del cientificismo de la época, era natural, como lo hicieron Marx y Engels, postular el isomorfismo entre las leyes económicas, sociales, históricas y las leyes de la Naturaleza. Al fin y al cabo, todas las ideas marxistas se fundamentan en los conceptos provenientes de la física clásica: continuidad, causalidad local, determinismo, objetividad."

30 Op. Cit., pág. 20. 
causal del paradigma científico moderno. El pasaje del nivel macrofísico a las entidades cuánticas, no se ha podido establecer todavía, ni siquiera utilizando el más puro y abstracto lenguaje matemático. Debido al margen de inexplicabilidad que tienen numerosos fenómenos en ambos mundos, no se han podido desechar ninguna de las teorías así como tampoco se han podido superar mutuamente. "La discontinuidad que se ha manifestado en el mundo cuántico se manifiesta en la estructura de niveles de Realidad, lo cual no impide que coexistan los dos mundos"3!.

Dentro de lo que podemos reconocer como las condiciones de posibilidad del cruce arte y ciencia que supone el bioarte, la cuestión del método resulta de capital importancia. Si pensamos en artistas invadiendo laboratorios debemos pensar en que además de las cuestiones epistemológicas que han flexibilizado los límites disciplinarios y que han reorganizado el universo del intercambio de conocimientos incorporando actores de lo más diversos, algo debe pasar en el orden de lo práctico, de la técnica, del arte de producir que hermana ambas esferas.

Desde el punto de vista de la biología, podemos rastrear el reconocimiento del uso de protocolos (no de métodos) basado en la aplicación continua de técnicas donde el azar se ha incorporado como parte de la explicación de los resultados; esta forma experimental de proceder es similar a la que utiliza el arte que viene sufriendo, desde hace más de medio siglo, transformaciones que dificultan su conceptualización. La apertura hacia nuevos horizontes de conocimiento, junto con la incorporación técnica que eso trae aparejado, ha dado lugar a la aparición de artistas -en condiciones de manipular los mismos elementos que utilizan los científicos- en los laboratorios.

El uso de protocolos - guías de experimentos- puede ser utilizado tanto por el arte como por la ciencia. En el caso de esta última, los mismos se combinan persiguiendo un fin epistemológico vinculado a la producción de un conocimiento nuevo, ya sea técnico, teórico o instrumental (bienes y servicios).

En el caso del arte, no existe la obligación de obtener un resultado innovador. Esto es algo que diferencia a ambas maneras de crear pero que, al mismo tiempo, las emparenta, dado que demuestra la ausencia de un determinismo en la metodología de trabajo utilizada. Es por ello también que puede suceder que el arte produzca conocimiento científico. Así como que la ciencia desarrolle una mirada estética sobre su propia práctica y pueda capitalizar esas reflexiones en función del desarrollo de una investigación más compleja.

Los márgenes de libertad que cada uno posee, y que construimos a partir de una visión moderna donde el arte y la ciencia constituyen esferas autónomas y aisladas de las demás, se potencian al momento del intercambio interdisciplinar que supone el bioarte -y que, desde la perspectiva de la historia de las ciencias de la vida, habilitó la biotecnología.

Son significativos los casos en los que obras de arte creadas en conjunto por artistas y científicos han producido giros en las investigaciones de laboratorio. Asimismo, los niveles de experimentación orientados por la curiosidad del artista han generado resultados inexplicables para la ciencia. Institu-

31 Op. Cit., pág. 24. 
ciones como Symbiotica (Australia), Biolab (Argentina), Incubator Art Lab (Canadá), BioArt Laboratories (Holanda) y artistas como Eduardo Kac (Brasil-EEUU), Paul Vanouse (EEUU), Marta de Menezes (Portugal), Joaquín Fargas (Argentina) entre muchísimos otros exponentes de la "vanguardia biológica"32, han puesto en jaque la construcción de conocimiento tradicional operando desde dentro de la lógica científica pero incorporando la mirada inmaculada de quien posee una formación alternativa.

Transcurrida más de una década desde su oficialización, es imposible pensar en la aparición de una nueva sensibilidad estética como el bioarte sin la construcción interdisciplinaria del campo de la biotecnología. En este contexto los artistas y los científicos pueden complementarse armoniosamente, sin anularse. Esto se debe a que, como hemos visto, las trayectorias de cada una son disímiles y están cargadas de imaginarios y maneras diferentes de intervenir en la realidad. No es fácil encontrar el equilibrio, pero la práctica del bioarte da muestras de que es posible pensar en una convivencia pacífica ente actores diversos que contagie y derribe, definitivamente, las fronteras histórico-políticas que han limitado el desarrollo de una forma de investigar más compleja, es decir, más humana.

\section{Bibliografía}

Abir-Am, Pnina Geraldine. "La biología molecular en el contexto de las culturas del Reino Unido, Francia y Estados Unidos" en La ciencia y sus culturas. Revista Internacional de Ciencias Sociales. Junio. N I 68. Organización de Estados Americanos. 200 I. Disponible en: http://www.oei.es/salactsi/abir.pdf Fecha de consulta: diciembre 2015.

Agar, Jon. "What happened in the sixties?" en The British Journal for the History of Science. 4 I (04). 2008. P. 567600. Disponible en: http://www.ucl.ac.uk/sts/staff/agar/documents/whathappenedinthesixties.pdf Fecha de consulta: diciembre 2015 .

Barinaga, Marcia. "Asilomar, vingt-cinq ans après" en La Recherche. L'actualité des sciences. N 332. P. 82. 01/06/2000. Disponible en: http://www.larecherche.fr/idees/livres/asilomar-vingt-cinq-ans-apres-0 I-06-2000-86943 Fecha de consulta: diciembre 2015 .

Bud, Robert. The uses of life: a history of biotechnology. Universidad de Cambridge. 1993.

Canguilhem, Georges. El conocimiento de la vida. Barcelona. Editorial Anagrama. 1976.

Costa, Flavia y Stubrin, Lucía. "Bioarte" en Kozak, Claudia (ed.) Tecnopoéticas argentinas. Archivo blando de arte y tecnología. Buenos Aires. Caja Negra Editora. 2012.

Facultad de Bioquímica y Ciencias Biológicas - Universidad Nacional del Litoral (2014) Diponible en: http://www. fbcb.unl.edu.ar/pages/estudios/carreras-de-grado/lic.-en-biotecnologia.php Fecha de consulta: diciembre 2015.

32 Stubrin, Lucía. "Aportes para el estudio de la vanguardia biológica latinoamericana" en Revista Nómadas. № 40. Bogotá. Universidad Central. Instituto de Estudios Sociales Contemporáneos. 20 I4, págs. I3 - |43. Disponible en: http://www.ucentral.edu.co/images/ editorial/nomadas/docs/40-8s-aportes-para-el-estudio-de-la-vanguardia.pdf Fecha de consulta: diciembre 20 I 5. 
Flusser, Vilém. "Creación científica y artística" [1982] en Artefacto. Pensamiento sobre la técnica. № 6. Buenos Aires: edición independiente. 2007. P. 75-77. Disponible en: http://www.revista-artefacto.com.ar/pdf_notas/I62. pdf Fecha de consulta: diciembre 2015.

"Arte vivo" [1988] en Artefacto. Pensamientos sobre la técnica. № 6. Buenos Aires: edición independiente. P. 77-80. 2007. Disponible en: http://www.revista-artefacto.com.ar/pdf_notas/I62.pdf Fecha de consulta: diciembre 2015.

Foucart, Jean. "Travail social et construction scientifique" en Pensée plurielle. 2008/3 n 19. p. 95-103. 2008.

García Canclini, Néstor. Diferentes, desiguales y desconectados. Mapas de la interculturalidad. Barcelona. Editorial Gedisa. 2004.

Kac, Eduardo. "El arte transgénico" en Leonardo Electronic Almanac. Vol. 6. № II. California. 1998. Disponible en: http://www.ekac.org/transgenico.html Fecha de consulta: diciembre 2015.

Kuhn, Thomas. La estructura de las revoluciones científicas. México. Fondo de Cultura Económica. 1986.

Latour, Bruno. Nunca fuimos modernos. Ensayo de antropología simétrica. Buenos Aires. Siglo XXI Editores. 2007.

Mitchell, Robert. Bioart and the vitality of media. Seattle. University of Washington Press. 2010.

Nicolescu, Basarab. La Transdisciplinariedad. Manifiesto. México. Edición: 7 Saberes. 1996.

Snow, Charles Percy. Las dos culturas [1959]. Introducción de Stefan Collini. Buenos Aires. Ediciones Nueva Visión. 2000.

Stubrin, Lucía. "Aportes para el estudio de la vanguardia biológica latinoamericana" en Revista Nómadas. № 40. Bogotá. Universidad Central. Instituto de Estudios Sociales Contemporáneos. 20|4. Pág. |3|-|43. Disponible en: http://www.ucentral.edu.co/images/editorial/nomadas/docs/40-8s-aportes-para-el-estudiode-la-vanguardia.pdf Fecha de consulta: diciembre 2015.

Vinck, Dominique. Pratiques de l'interdiciplinarité. Grenoble. Pub. 2000. 